\title{
A Framework For Classifying Semantic Relationships
}

\author{
Amaal Saleh Hassan Al Hashimy \\ Computer Science Department, Sultan Qaboos University \\ Muscat, Oman \\ amaalh@squ.edu.om
}

\author{
Narayanan Kulathuramaiyer \\ Knowledge Systems Department, University of UNIMAS \\ Sarawak, Malaysia \\ nara@fit.unimas.my
}

\begin{abstract}
Recently, the NLP community has shown a renewed interest in lexical semantics in the extent of automatic recognition of semantic relationships between pairs of words in text. Lexical semantics has become increasingly important in many natural language applications, this approach to semantics is concerned with psychological facts associated with meaning of words and how these words can be connected in semantic relations to build ontologies that provide a shared vocabulary to model a specified domain. And represent a structural framework for organizing information across fields of Artificial Intelligence (AI), Semantic Web, systems engineering and information architecture. But current systems mainly concentrate on classification of semantic relations rather than to give solutions for how these relations can be created [14]. At the same time, systems that do provide methods for creating the relations tend to ignore the context in which the conceptual relationships occur. Furthermore, methods that address semantic (non-taxonomic) relations are yet to come up with widely accepted ways of enhancing the process of classifying and extracting semantic relations.
\end{abstract}

In this research we will focus on the learning of semantic relations patterns between word meanings by taking into consideration the surrounding context in the general domain. We will first generate semantic patterns in domain independent environment depending on previous specific semantic information, and a set of input examples. Our case of study will be causation relations. Then these patterns will classify causation in general domain texts taking into consideration the context of the relations, and then the classified relations will be used to learn new causation semantic patterns.

Keywords-lexical semantics; semantic relationship; causation; ontology.

\section{INTRODUCTION}

Lexical semantics defined as the field that is concerned with the study of what individual lexical items mean, why they mean what they do, and how we can represent all of this [10]. Its concern lies within the changes of word meaning, identification, classification, and explanation of semantic changes [13]. It provides a wealth of theoretical proposals and empirical descriptions over many fields of applied research, like computational linguistics [24], language acquisition and learning [25] [12] [23], and how dictionaries are created, the science of lexicography [6]. A very interesting development with regards to lexical semantics is WordNet [20], which is a lexical database that attempts to model cognitive processes.

However the semantic relations are not always clear [18], some patterns can be vague as they represent various relations, e.g. "Hasan's car", and "Hasan's brother", has the same pattern but holds two relations, possession, and kinship respectively.
Another source of difficulty comes from the nature of the approaches used. Systems based on a purely syntactic approach demands manual intervention for analysis in preparing intermediate constructs for extracting these relationships [17] [2]. Besides that, syntactic patterns are also always limited in their representation power to a specific relation type, and as such are restricted to the set of processed examples [4]. Probabilistic approaches on the other hand [26] require the use of special kinds of resources like temporal data. These sources however do not always exist for all domains.

Existing systems mainly concentrate on classification of relations rather than to give solutions for how these relations can be created [1]. At the same time, systems that do provide methods for creating the relations tend to ignore the context in which the conceptual relationships occur. Furthermore, methods that address semantic relations of non-taxonomic type, are yet to come up with widely accepted ways of enhancing the process of classifying and extracting semantic relations.

In order to avoid this limitation we propose the use of semantic constrains to enforce such distinctions in discovering the relation; this is expected to yield better formulated patterns that actually represent the semantic constrains of the relation. Doing this in an automatic way (automatic recognition of semantic relationships) may include many potential methodologies and can be used in many NLP applications at the same time. NLP methods and theories in general implement some higher goals as a basis for automating the modeling of human agents' beliefs and desires in supporting their knowledge sharing [1].

\section{LITERATURE REVIEW}

In the early days of lexical semantics, (before the 1950s) the nature of lexical meaning was concerned with the psychological conception where the meaning of the word was considered as mental entities [22]. Subsequently, structuralism (Ferdinand \& Saussure, 1959) movement visualized language as an independent intra-linguistic system of relations between words, organized on the basis of lexical fields [8]. Based on the notion of structuralism of Paradis [21], we adopt a cognitive approach for conceptually assigning the meaning of the lexical units. We therefore consider aspects of contextual meaning modulation that is relevant for a certain research task by integrating semantics, pragmatics and grammar [13], [21]. The words in this approach are mapped directly to conceptual structure where these structures are evoked according to usage and the context [19] [7] [32].

Villaverde, Persson, Godoy, and Amandi [33] proposed a technique for the discovery of non-taxonomic relationships and 